\title{
SPECTROSCOPIC OBSERVATIONS OF THE SANDULEAK-SEGGEWISS STARS IN NGC 6231
}

\author{
L. A. MILONE
}

Observatorio Astronómico and IMAF, Universidad Nacional, Córdoba, Argentina

\begin{abstract}
Sanduleak and independently Seggewiss discovered a very red star near the open cluster NGC 6231. Bessell and collaborators concluded it to be a variable of the $\mathrm{R}$ Coronae Borealis type.

A pair of spectrograms of this object were obtained in July 1970. The spectrograph dispersion in the range of $3800-4500 \AA$ is around $80 \AA \mathrm{mm}^{-1}$ and in the range of $4500-7000 \AA 155 \AA \mathrm{mm}^{-1}$.

An outstanding feature of the spectrograms is their 'veiled' aspect, making it rather difficult to observe the lines. We observe the $4226 \AA$ of $\mathrm{Ca} \mathrm{I}, \mathrm{H}$ and $\mathrm{K}$ of Ca II, $4030 / 4034 \AA$ of $\mathrm{Mn}$ I, the G band; and several lines belonging to the $\mathrm{Fe}_{\mathrm{I}}$ multiplets. The $\mathrm{H} \alpha$ and $\mathrm{H} \beta$ lines are clearly seen in emission. $\mathrm{H} \delta$ is absent or very faint. A spectrogram of RY Sagitarii taken in July 16, 1971, when the visual magnitude of the star was around 4 mag. below its maximum light, shows some similarities with the spectrograms of the Sanduleak-Seggewiss star, e.g. they do not show the ultraviolet $\mathrm{CN}$ absorption bands and in the spectra of both stars a line is seen at approximately $3896 \AA$, which may be $\mathrm{H} 8$.

If we accept that the star belongs to NGC 6231, its distance modulus is 11.5. Its intrinsic color may be assumed to be close to $(B-V)_{0}=1.0(\mathrm{G}$ or $\mathrm{K}$ star $)$. The observed color is $(B-V)=3.5$. Adopting $\mathrm{R}=3$, the visual absorption is 7.5 . As the visual magnitude of the star is $V=12.5$, its absolute magnitude becomes -6.5 .

Details will be published elsewhere.
\end{abstract}

